\title{
As Simetrias de Lie de um Pião
}

\author{
The Lie symmetries of a Top
}

\author{
Cláudio H. C. Costa Basquerotto*1, Edison Righetto ${ }^{2}$, Samuel da Silva ${ }^{1}$ \\ ${ }^{1}$ Universidade Estadual Paulista, Departamento de Engenharia Mecânica, Ilha Solteira, SP, Brasil. \\ ${ }^{2}$ Universidade Estadual Paulista, Departamento de Matemática, Ilha Solteira, SP, Brasil.
}

Recebido em 13 de Setembro, 2017. Revisado em 07 de Outubro, 2017. Aceito em 13 de Outubro, 2017

\begin{abstract}
A existência de simetrias em equações diferenciais pode gerar transformações em variáveis dependentes e independentes que facilitam a integração destas equações. Em especial, Sophus Lie desenvolveu no século XIX uma forma de extração de simetrias que podem ser usadas efetivamente para revelar as integrais primeiras, ou seja, as constantes de movimento, que muitas vezes podem estar escondidas. Estes invariantes podem em algumas situações ser identificados pelo teorema de Noether ou a partir de manipulações das próprias equações com transformações de Lie. Nos cursos iniciais de mecânica clássica, apesar de todo o formalismo em cima dos teoremas de conservação de energia e momento linear/angular, a relação disto com a existência de possíveis simetrias de Lie não é destacada de forma clara e objetiva. Neste sentido, o presente artigo busca apresentar uma introdução às simetrias de Lie usando uma linguagem acessível para um aluno de graduação de física, matemática ou engenharia com domínio básico em fundamentos de cálculo com várias variáveis. Para ilustrar a abordagem, considera-se um problema clássico de mecânica considerando um pião em regime de movimento com precessão estacionária. A partir das equações de movimento obtidas, as simetrias de Lie são identificadas e usadas na transformação para a redução da ordem. As integrais primeiras são obtidas a partir deste resultado com o Teorema de Noether, mostrando que neste exemplo e condição as simetrias de Lie também são simetrias de Noether. Por fim, a resolução das equações de movimento podem ser feitas usando funções elípticas de Jacobi para a obtenção dos ângulos de precessão, nutação e spin nas condições apresentadas.
\end{abstract}

Palavras-chave: Simetrias de Lie. Teorema de Noether. Pião simétrico. Constantes de movimento. Funções elípticas de Jacobi.

The existence of symmetries in differential equations can generate transformations of dependent and independent variables that facilitate the integration of these equations. In the nineteenth century, Sophus Lie developed a method of extracting symmetries that can be used effectively to reveal first integrals of a differential equation. These invariants can in some situations be identified by the Noether theorem or from manipulating the equations themselves with Lie transformations. Despite the formalism over conservation theorems for energy and linear/angular momentum, initial courses in classical mechanics often do not clearly or objectively highlight the relationship between conservation laws and the existence of possible Lie symmetries. For this reason, we seek to present an introduction to Lie symmetries using a language accessible to a graduate student in physics, mathematics, or engineering with basic mastery of classical mechanics in several variables. In order to illustrate the approach, we consider the classical problem of a spinning top with stationary precession. From the equations of motion, the Lie symmetries are identified and used in a transformation that results in an order reduction. The first integrals are obtained from this result using the Noether theorem, and we illustrate that the Lie symmetries for this problem are also Noether symmetries. Finally, the solution to the equations of motion are written using Jacobi elliptic functions, and from this we obtain the precession, nutation and spin angles under the presented conditions.

Keywords: Lie symmetries. Noether theorem. Symmetric top. Motion constants. Jacobi elliptic functions.

\section{Introdução}

Muitos problemas encontrados em ciência podem ser descritos por equações diferenciais que precisam ser resolvidas ou aproximadas por métodos analíticos ou numéricos de integração $[1]$. No século XIX, o norueguês Sophus Lie apresentou uma alternativa visando solucionar equa-

*Endereço de correspondência: cbasquerotto@ymail.com ções diferenciais usando simetrias que podem se associar com invariantes ou quantidades conservadas [4,5].

Uma simetria de um sistema de equações diferenciais é um conjunto de transformações que mapeiam qualquer solução para outra solução do sistema, mantendo-a invariante 6.7]. Tais transformações são grupos que dependem de parâmetros contínuos e consistem em transformações pontuais, também conhecidas como simetrias pontuais, atuando no espaço do sistema de variáveis dependen- 
tes e independentes, bem como em todas as derivadas das variáveis dependentes [8]. Exemplos elementares de grupos de Lie incluem as translações, rotações e escalonamento que podem ser feitos corriqueiramente em algumas equações diferenciais. Porém, outras simetrias não triviais também são possíveis de existirem.

Assim, o conhecimento de grupos de transformações de Lie de um sistema de equações diferenciais pode ser usado, por um lado, para reduzir a ordem ou, em alguns casos, reduzir as próprias equações diferenciais, tanto ordinárias quanto parciais, para uma quadratura ${ }^{1}$ 9 10 . As simetrias têm sido usadas para obter soluções dos mais diferentes tipos de problemas, como por exemplo, vorticidade em dinâmica de fluidos 11, equação do calor [12 14], equação da onda 15, 16], mecânica do contínuo envolvendo plasticidade 17], problemas de elasticidade em barras, vigas e placas [18, 19], teoria de campos a temperatura finita e teoria cinética relativística 20], entre outros.

As relações entre simetria e invariância são fundamentais na física e aparecem em todas as áreas a partir de princípios de conservação 21]. Um caso particular, é a obtenção de leis de conservação a partir da aplicação do Teorema de Noether ${ }^{2}$ em sistemas de equações diferenciais variacionais descritos por uma lagrangiana [22 24]. Em um único artigo, Noether publicou suas descobertas envolvendo a relação crucial entre simetrias e constantes se conservando, bem como o impacto de simetrias nas equações de movimento. Vários autores mostram que algumas classes de equações diferenciais geradoras de simetria de Lie também são simetrias de Noether, ou seja, estão associadas com a propriedade bem conhecida envolvendo os princípios de conservação de energia, conservação de momento linear e conservação de momento angular 25 27]. Sabe-se que uma simetria de Lie também é uma simetria de Noether se há a conservação da ação integral (lagrangiana) [28]. No entanto, existem casos que uma simetria de Noether não é uma simetria de Lie 29]. Os contra-exemplos mais comuns são dados por grupos de transformação de escala [30]. O teorema de Noether, enunciado no início do século XX, diz respeito à invariância de um problema variaciona ${ }^{3}$ sob a ação de um grupo de Lie com um número finito de geradores infinitesimais independentes, que é situação típica na mecânica clássica e na relatividade especial 31. Neste teorema, Emmy Noether formulou com completa

\footnotetext{
$\overline{{ }^{1} \text { Por quadratura se entende }}$ uma integral com resultado inverso conhecido por funções elementares ou especiais, por exemplo funções elípticas de Jacobi.

${ }^{2} \mathrm{O}$ trabalho magistral de Emmy Noether, 53, completa 100 anos em 2018.

${ }^{3}$ As equações da mecânica clássica e relativística e da física são obtidas exigindo que uma integral de ação associada a uma lagrangiana que descreva o sistema, seja extrema. Tais equações são chamadas equações variacionais ou equações de Euler-Lagrange. Pode-se dizer que elas derivam de um princípio variacional, também chamado de princípio de ação. Elas expressam a anulação da derivada variacional, também denominada derivada de Euler-Lagrange ou diferencial de Euler-Lagrange da lagrangiana.
}

generalidade a correspondência entre as simetrias de um problema variacional e as leis de conservação para as equações variacionais associadas 32,33 .

Neste sentido, este artigo busca determinar as simetrias de Lie associadas com as equações diferenciais ordinárias que descrevem o movimento de um pião simétrico em movimento de precessão estacionária e aplicá-las ao teorema de Noether para obtenção de constantes de movimento. Provada a origem da conservação de energia e momentos, pode-se determinar a solução analítica das equações do movimento através da solução por quadraturas por meio de funções elípticas de Jacobi, que foram recentemente discutidas em Lemos (2017) 34]. Em seu trabalho, Lemos (2017) discute a importância de se utilizar as funções elípticas para a solução de vários problemas interessantes encontrados na física, embora estas não sejam muito populares nos dias atuais.

Este trabalho está organizado em seis seções. Primeiramente é feita uma breve introdução sobre as equações de movimento usadas e uma fundamentação matemática básica envolvendo simetrias de Lie. Importante destacar que as simetrias de Lie são apresentadas com uma notação e com base em conhecimentos básicos de cálculo de várias variáveis que se espera de um aluno no segundo ano de um curso de ciências exatas. Posteriormente, as simetrias de Lie do pião simétrico com um ponto fixo são extraídas. Com isso, é feita a aplicação das simetrias de Lie no teorema de Noether para a indentificação das constantes de movimento e posterior redução de ordem para a determinação da solução analítica do problema envolvendo a manipulação com funções elípticas de Jacobi, que ainda não são amplamente usadas em um curso de mecânica clássica 35, 36]. Por fim, as considerações finais são apresentadas.

\section{Pião Simétrico com um Ponto Fixo}

A figura (1) representa um pião simétrico com massa $m$ que está fixo em $O$. Para se parametrizar o movimento do pião no espaço, deve-se fazer uma sequência de três rotações consecutivas em torno de três eixos diferentes, utilizando os ângulos de Euler. Primeiramente, toma-se como referência inercial, $\mathcal{I}$, o eixo $(X, Y, Z)$ e $\{\hat{i}, \hat{j}, \hat{k}\}$ fixo em $O$. A primeira rotação é em torno do eixo $Z \equiv z_{1}$ com velocidade angular $\dot{\psi}$ com um ângulo de precessão $\psi$ no sistema móvel $\mathcal{B}_{1} \operatorname{com}\left(x_{1}, y_{1}, z_{1}\right)$ e $\left\{\hat{i}_{1}, \hat{j}_{1}, \hat{k}_{1}\right\}$. O segundo giro é feito em torno de $x_{1} \equiv x_{2}$ no sentido positivo, com velocidade angular $\dot{\theta}$ e com um ângulo de nutação $\theta$ no sistema móvel $\mathcal{B}_{2} \operatorname{com}\left(x_{2}, y_{2}, z_{2}\right)$ e $\left\{\hat{i}_{2}, \hat{j}_{2}, \hat{k}_{2}\right\}$. Por fim, é feita a última rotação em torno de $z_{2} \equiv z$ com uma velocidade angular $\dot{\phi}$ e um ângulo $\phi$ no sistema móvel $\mathcal{B}_{3} \operatorname{com}(x, y, z)$ e $\left\{\hat{i}_{3}, \hat{j}_{3}, \hat{k}_{3}\right\}$. O centro de massa do pião é representado pelo ponto $A$ e a distância do ponto $O$ até este ponto ao longo do eixo $z$ é $\ell 35,37$. 


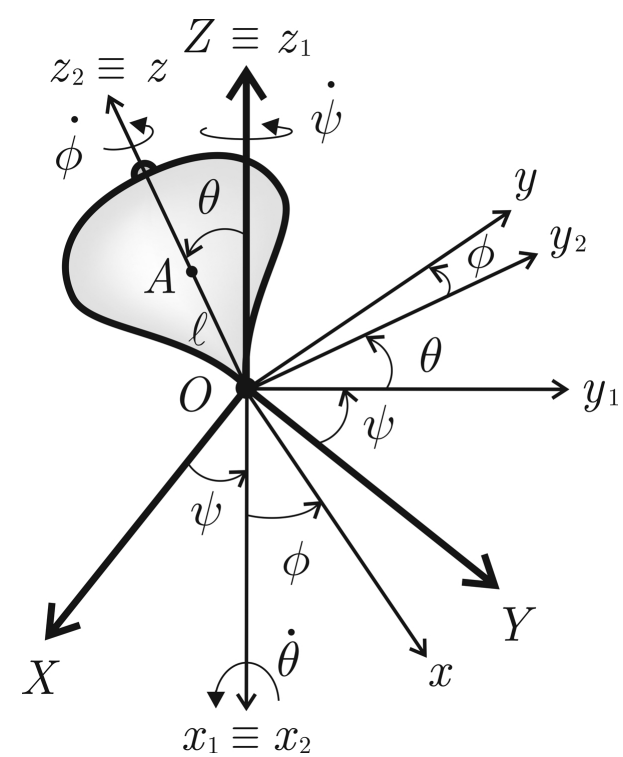

Figura 1: Pião simétrico com um ponto fixo.

A partir da figura (1) é possível descrever a velocidade angular do pião, $\mathcal{B}_{2} \omega$, no sistema $\mathcal{B}_{2}$ coincidente com os eixos de simetria [38]:

$$
\begin{array}{r}
\mathcal{B}_{2} \boldsymbol{\omega}={ }_{\mathcal{B}_{2}} \dot{\boldsymbol{\Psi}}+{ }_{\mathcal{B}_{2}} \dot{\boldsymbol{\Theta}}+{ }_{\mathcal{B}_{2}} \dot{\boldsymbol{\Phi}}= \\
\dot{\theta} \hat{i}_{2}+\dot{\psi} \operatorname{sen} \theta \hat{j}_{2}+(\dot{\phi}+\dot{\psi} \cos \theta) \hat{k}_{2}
\end{array}
$$

sendo ${ }_{\mathcal{B}_{2}} \dot{\boldsymbol{\Psi}}$ o vetor velocidade angular de precessão, ${ }_{\mathcal{B}_{2}} \dot{\Theta}$ o vetor velocidade angular de nutação e $\mathcal{B}_{2} \dot{\boldsymbol{\Phi}}$ o vetor velocidade angular de rotação própria do pião, todos representados na base $\mathcal{B}_{2}$. A lagrangiana pode ser obtida por 37:

$$
\mathcal{L}=\mathcal{T}-\mathcal{V}
$$

sendo $\mathcal{T}$ a energia cinética dada por:

$$
\mathcal{T}=\frac{1}{2} \mathcal{B}_{2} \omega \cdot \mathbb{I} \cdot \mathcal{B}_{2} \omega
$$

e II o tensor de inércia do pião de massa $m$ calculado com relação aos eixos principais $x_{2}, y_{2}$ e $z_{2}$, dado por:

$$
\mathbb{I}=\left|\begin{array}{ccc}
I_{x_{2}} & 0 & 0 \\
0 & I_{y_{2}} & 0 \\
0 & 0 & I_{z_{2}}
\end{array}\right|
$$

sendo $I_{x_{2}}, I_{y_{2}}$ e $I_{z_{2}}$ componentes do tensor de inércia com relação aos eixos $x_{2}, y_{2}$ e $z_{2}$ respectivamente. Considerando que o pião é simétrico, então $I_{x_{2}} \equiv I_{y_{2}}=I$, com isso:

$$
\mathbb{I}=\left|\begin{array}{ccc}
I & 0 & 0 \\
0 & I & 0 \\
0 & 0 & I_{z_{2}}
\end{array}\right|
$$

Uma vez calculada a velocidade angular do pião, a energia cinética fica descrita como:

$$
\mathcal{T}=\frac{1}{2} I\left(\dot{\theta}^{2}+\dot{\psi}^{2} \operatorname{sen}^{2} \theta\right)+\frac{1}{2} I_{z_{2}}(\dot{\phi}+\dot{\psi} \cos \theta)^{2}
$$

e a energia potencial, $\mathcal{V}$ :

$$
\mathcal{V}=m g \ell \cos \theta
$$

sendo $g$ a aceleração da gravidade.

Com isto:

$\mathcal{L}=\frac{1}{2} I\left(\dot{\theta}^{2}+\dot{\psi}^{2} \operatorname{sen}^{2} \theta\right)+\frac{1}{2} I_{z_{2}}(\dot{\phi}+\dot{\psi} \cos \theta)^{2}-m g \ell \cos \theta$

Assim, com o uso das equações de Euler-Lagrange são obtidas as equações de movimento do pião:

$$
\begin{aligned}
& I\left(\ddot{\theta}-\dot{\psi}^{2} \operatorname{sen} \theta \cos \theta\right)+I_{z_{2}}\left(\dot{\psi}^{2} \operatorname{sen} \theta \cos \theta+\dot{\phi} \dot{\psi} \operatorname{sen} \theta\right) \\
& -m g \ell \operatorname{sen} \theta=0 \\
& I(\ddot{\psi} \operatorname{sen} \theta+2 \dot{\psi} \dot{\theta} \cos \theta)-I_{z_{2}} \dot{\theta}(\dot{\psi} \cos \theta+\dot{\phi})=0 \\
& I_{z_{2}}(\ddot{\phi}+\ddot{\psi} \cos \theta-\dot{\psi} \dot{\theta} \operatorname{sen} \theta)=0
\end{aligned}
$$

Assumindo que as velocidades angulares de precessão $\dot{\psi}$ e $\dot{\phi}$ são constante, pode-se modificar a eq. (7) e obter as equações do movimento para o pião para a condição particular considerada:

$$
\begin{aligned}
& I\left(\ddot{\theta}-\dot{\psi}^{2} \operatorname{sen} \theta \cos \theta\right)+I_{z_{2}}\left(\dot{\psi}^{2} \operatorname{sen} \theta \cos \theta+\dot{\phi} \dot{\psi} \operatorname{sen} \theta\right) \\
& -m g \ell \operatorname{sen} \theta=0 \\
& I(2 \dot{\psi} \dot{\theta} \cos \theta)-I_{z_{2}} \dot{\theta}(\dot{\psi} \cos \theta+\dot{\phi})=0 \\
& I_{z_{2}}(\dot{\psi} \dot{\theta} \operatorname{sen} \theta)=0
\end{aligned}
$$

As equações do movimento obtidas são equações diferenciais ordinárias não-lineares [39]. Para facilitar o cálculo das simetrias de Lie, é interessante escrever essas equações na forma $\mathcal{F}_{i}=0$, com $i=1,2,3$. Com isso, as equações de movimento ficam:

$$
\begin{aligned}
& \mathcal{F}_{1}=I\left(\ddot{\theta}-\dot{\psi}^{2} \operatorname{sen} \theta \cos \theta\right) \\
& +I_{z_{2}}\left(\dot{\psi}^{2} \operatorname{sen} \theta \cos \theta+\dot{\phi} \dot{\psi} \operatorname{sen} \theta\right)-m g \ell \operatorname{sen} \theta=0 \\
& \mathcal{F}_{2}=I(2 \dot{\psi} \dot{\theta} \cos \theta)-I_{z_{2}} \dot{\theta}(\dot{\psi} \cos \theta+\dot{\phi})=0 \\
& \mathcal{F}_{3}=I_{z_{2}}(\dot{\psi} \dot{\theta} \operatorname{sen} \theta)=0
\end{aligned}
$$

Nota-se também que as variáveis $\psi$ e $\phi$ não aparecem explicitamente na lagrangiana sendo, portanto, variáveis cíclicas. A existência de uma variável cíclica indica que os momentos generalizados correspondentes são constantes [40]. Ao se determinar as simetrias de Lie, encontram-se geradores infinitesimais correspondentes à essas mesmas variáveis que quando aplicados ao teorema de Noether revelam também estes invariantes, como será mostrado a seguir.

\section{Simetrias de Lie}

Esta seção busca apresentar os fundamentos básicos envolvidos na extração de simetrias de Lie a partir de sistemas de equações diferenciais. O assunto aqui abordado é amplamente disponível em excelentes livros textos como $30,41,42$. 


\subsection{Geradores infinitesimais}

De forma geral, assumindo uma variável genérica $q=$ $q(t)$, um grupo de transformação envolvendo as variáveis dependente $q$ e independente $t$ a um parâmetro contínuo $\varepsilon \in \mathbb{R}$ pode ser feito para obter novas variáveis $\bar{t}$ e $\bar{q}$ a partir de 30,41, 42]:

$$
\bar{t}=\alpha(t, q, \varepsilon), \quad \bar{q}=\mu(t, q, \varepsilon)
$$

sendo $\alpha$ e $\mu$ funções analíticas que, normalmente, são desconhecidas e que realizam estas transformações. Podese expandir $\bar{t}$ e $\bar{q}$ usando séries de MacLaurin em torno de $\varepsilon$ através de:

$$
\bar{t} \approx t+\varepsilon\left(\left.\frac{\partial \alpha}{\partial \varepsilon}\right|_{\varepsilon \rightarrow 0}\right), \quad \quad \bar{q} \approx q+\varepsilon\left(\left.\frac{\partial \mu}{\partial \varepsilon}\right|_{\varepsilon \rightarrow 0}\right)
$$

desde que $\varepsilon \rightarrow 0$ constitui a identidade do grupo. Definindo novas funções infinitesimais descritas por:

$$
\xi(t, q)=\left.\frac{\partial \alpha}{\partial \varepsilon}\right|_{\varepsilon \rightarrow 0}, \quad \eta_{q}(t, q)=\left.\frac{\partial \mu}{\partial \varepsilon}\right|_{\varepsilon \rightarrow 0}
$$

pode-se reescrever as expressões da equação 11 como:

$$
\bar{t} \approx t+\varepsilon \xi(t, q), \quad \bar{q} \approx q+\varepsilon \eta_{q}(t, q)
$$

Contudo, além das mudanças nas variáveis $t$ e $q$, uma extensão para as derivadas de alta ordem $\dot{q}$ e $\ddot{q}$ devem ser obtidas, conhecidos como prolongamentos de ordem superior.

\subsection{Prolongamento das transformações e seus geradores}

Uma vez que as transformações com os geradores infinitesimais será feita em uma equação diferencial é necessário se prolongar as transformações nas respectivas derivadas $43[46]$. A aproximação por $\dot{\bar{q}}$ fica sendo:

$$
\dot{\bar{q}}=\frac{d \bar{q}}{d \bar{t}}
$$

sendo $d \bar{q}=d q+\varepsilon d \eta_{q}$ e $d \bar{t}=d t+\varepsilon d \xi$. Derivando estes termos com relação a $t$ :

$$
\frac{d \bar{q}}{d t}=\dot{q}+\varepsilon \mathcal{D}_{t}\left(\eta_{q}\right), \quad \frac{d \bar{t}}{d t}=1+\varepsilon \mathcal{D}_{t}(\xi)
$$

sendo $\mathcal{D}_{t}$ a derivada total, dada por:

$$
\mathcal{D}_{t}=\frac{\partial}{\partial t}+\dot{q} \frac{\partial}{\partial q}+\ddot{q} \frac{\partial}{\partial \dot{q}}
$$

pela definição anterior, sabe-se que $\varepsilon \rightarrow 0$ e com o auxílio da regra do binomial ${ }^{4}$

$$
\begin{aligned}
& \dot{\bar{q}}=\frac{\dot{q}+\varepsilon \mathcal{D}_{t}\left(\eta_{q}\right)}{1+\varepsilon \mathcal{D}_{t}(\xi)} \approx\left\{\dot{q}+\varepsilon \mathcal{D}_{t}\left(\eta_{q}\right)\right\}\left\{1-\varepsilon \mathcal{D}_{t}(\xi)\right\}= \\
& \dot{q}-\dot{q} \varepsilon \mathcal{D}_{t}(\xi)+\varepsilon \mathcal{D}_{t}\left(\eta_{q}\right)-\varepsilon^{2} \mathcal{D}_{t}\left(\eta_{q}\right) \mathcal{D}_{t}(\xi) \approx \dot{q}+\varepsilon \beta^{(1)}
\end{aligned}
$$

$\overline{4(1+x)^{\lambda}=1+\lambda x+\frac{\lambda(\lambda-1)}{2}} x^{2}+\cdots$ como pode-se observar, na penúltima passagem há uma simplificação, já que $\varepsilon \approx 0$ consequentemente $\varepsilon^{2}=0$. $\mathrm{O}$ termo $\beta^{(1)}$ pode ser escrito como:

$$
\begin{aligned}
& \beta^{(1)}(t, q, \dot{q})=\mathcal{D}_{t}\left(\eta_{q}\right)-\dot{q} \mathcal{D}_{t}(\xi) \\
& =\frac{\partial \eta_{q}}{\partial t}+\left(\frac{\partial \eta_{q}}{\partial q}-\frac{\partial \xi}{\partial t}\right) \dot{q}-\frac{\partial \xi}{\partial q}(\dot{q})^{2}
\end{aligned}
$$

que é o primeiro prolongamento.

Em sistemas de equações diferenciais com termos de alta ordem, deve-se estender essa ideia para essas variáveis. Com isso, pode-se escrever $\ddot{\bar{q}}$ através do campo vetorial $\gamma=\left\{\begin{array}{ll}\xi & \eta_{q}\end{array}\right\}^{T}$ e $\varepsilon$ através do mesmo procedimento realizado anteriormente.

$$
\begin{gathered}
\ddot{\bar{q}}=\frac{d \dot{\bar{q}}}{d \bar{t}}=\frac{\mathcal{D}_{t}\left(\dot{q}+\varepsilon \beta^{(1)}\right)}{\mathcal{D}_{t}(t+\varepsilon \xi)}=\frac{\ddot{q}+\varepsilon \mathcal{D}_{t}\left(\beta^{(1)}\right)}{1+\varepsilon \mathcal{D}_{t}(\xi)} \approx \\
\left\{\ddot{q}+\varepsilon \mathcal{D}_{t}\left(\beta^{(1)}\right)\right\}\left\{1-\varepsilon \mathcal{D}_{t}(\xi)\right\} \approx \ddot{q}+\varepsilon \beta^{(2)}
\end{gathered}
$$

sendo:

$$
\begin{aligned}
& \beta^{(2)}(t, q, \dot{q}, \ddot{q})=\mathcal{D}_{t}\left(\beta^{(1)}\right)-\ddot{q} \mathcal{D}_{t}(\xi) \\
& =\frac{\partial^{2} \eta_{q}}{\partial t^{2}}+\left(2 \frac{\partial^{2} \eta_{q}}{\partial t \partial q}-\frac{\partial^{2} \xi}{\partial t^{2}}\right) \dot{q} \\
& +\left(\frac{\partial^{2} \eta_{q}}{\partial q^{2}}-2 \frac{\partial^{2} \xi}{\partial t \partial q}\right)(\dot{q})^{2}-\frac{\partial^{2} \xi}{\partial q^{2}}(\dot{q})^{3} \\
& +\left(\frac{\partial \eta_{q}}{\partial q}-2 \frac{\partial \xi}{\partial t}\right) \ddot{q}-3 \frac{\partial \xi}{\partial q} \dot{q} \ddot{q}
\end{aligned}
$$

o prolongamento de segunda ordem [42]. De forma geral um prolongamento de ordem $\mathrm{k}$ é dado por 42]:

$$
\begin{aligned}
& \beta^{(\mathrm{k})}(t, q, \dot{q}, \ddot{q}, \ldots, q) \\
& =\left(\mathcal{D}_{t}\right)^{\mathrm{k}} \eta_{q}-\sum_{j=1}^{\mathrm{k}} \frac{\mathrm{k} !}{(\mathrm{k}-j) ! j !}{ }^{(\mathrm{k}-j+1)}\left(\mathcal{D}_{t}\right)^{j} \xi
\end{aligned}
$$

sendo $\mathrm{k}=1,2,3, \ldots$.. Lembrando que $q$ pode ser $\theta, \phi$ ou $\psi$ no problema considerado neste artigo.

\subsection{Teorema de Lie}

Após calcular o prolongamento das transformações e considerando agora as coordenadas originais $\theta, \psi$ e $\phi$, uma vez conhecido o campo vetorial $\gamma=\left\{\begin{array}{llll}\xi & \eta_{\theta} & \eta_{\psi} & \eta_{\phi}\end{array}\right\}^{T}$, um gerador infinitesimal de simetria pode ser obtido a partir de 47:

$$
\mathcal{X}=\left\{\begin{array}{c}
\xi \\
\eta_{\theta} \\
\eta_{\psi} \\
\eta_{\phi}
\end{array}\right\} \cdot\left\{\begin{array}{c}
\frac{\partial}{\partial t} \\
\frac{\partial}{\partial \theta} \\
\frac{\partial}{\partial \psi} \\
\frac{\partial}{\partial \phi}
\end{array}\right\}=\xi \frac{\partial}{\partial t}+\eta_{\theta} \frac{\partial}{\partial \theta}+\eta_{\psi} \frac{\partial}{\partial \psi}+\eta_{\phi} \frac{\partial}{\partial \phi}
$$

e aplicando o operador de segunda ordem $\mathcal{U}^{\prime \prime}$ para estas variáveis 48]:

$$
\mathcal{U}^{\prime \prime}=\beta^{(1)}\left(\frac{\partial}{\partial \dot{\theta}}+\frac{\partial}{\partial \dot{\psi}}+\frac{\partial}{\partial \dot{\phi}}\right)+\beta^{(2)} \frac{\partial}{\partial \ddot{\theta}}
$$


Tabela 1: Geradores infinitesimais da equação (9).

\begin{tabular}{lll}
\hline & $\xi$ & $\eta_{q}$ \\
\hline $\mathcal{X}_{1}=\frac{\partial}{\partial t}$ & 1 & 0 \\
$\mathcal{X}_{2}=\frac{\partial}{\partial \psi}$ & 0 & 1 \\
$\mathcal{X}_{3}=\frac{\partial}{\partial \phi}$ & 0 & 1 \\
\hline
\end{tabular}

Com isso, a condição de Lie é dada por:

$$
\begin{gathered}
\left(\mathcal{U}^{\prime \prime}+\mathcal{X}\right) \mathcal{F}_{i}=0 \\
\left(\mathcal{U}^{\prime \prime}+\mathcal{X}\right) \mathcal{F}_{i}=\xi \frac{\partial \mathcal{F}_{i}}{\partial t}+\eta_{\theta} \frac{\partial \mathcal{F}_{i}}{\partial \theta}+\eta_{\psi} \frac{\partial \mathcal{F}_{i}}{\partial \psi}+\eta_{\phi} \frac{\partial \mathcal{F}_{i}}{\partial \phi} \\
+\beta^{(1)}\left(\frac{\partial}{\partial \dot{\theta}}+\frac{\partial}{\partial \dot{\psi}}+\frac{\partial}{\partial \dot{\phi}}\right)+\beta^{(2)} \frac{\partial}{\partial \ddot{\theta}}=0
\end{gathered}
$$

sendo $\mathcal{F}_{i}$ o sistema de equações dado pela equação $(9)$ com $i=1,2,3$.

O critério infinitesimal de invariância, descrito pela equação (24), envolve $t, \theta, \psi$ e $\phi$, e as derivadas de $\theta$, $\psi$ e $\phi$ com respeito a $t$, assim como as variáveis $\xi, \eta_{\theta}$, $\eta_{\psi}$ e $\eta_{\phi}$. Depois de eliminar toda a dependência das derivadas envolvendo $\theta, \psi$ e $\phi$, pode-se equacionar os coeficientes das derivadas parciais remanescentes de $\theta$, $\psi$ e $\phi$ à zero. Com isso, pode-se ter como resultado um grande número de equações diferenciais parciais para determinar as funções $\xi, \eta_{\theta}, \eta_{\psi}$ e $\eta_{\phi}$. Estas equações são conhecidas como equações determinantes para um grupo de simetrias de um determinado sistema [30]. A determinação das soluções destas equações determinantes pode ser feita através da utilização de alguns pacotes de manipulação simbólica, como o wxMaxima, Mathematica (Sym 49] e/ou MathLie [41]), Maple (DETools e/ou PDETools), entre outros.

\subsection{Aplicação das simetrias de Lie nas equações do Pião}

Aplicando a condição de Lie, dada pela equação (24), no sistema de equações (9), é possível obter as seguintes equações determinantes: $\frac{\partial \eta_{\psi}}{\partial \psi}=0, \frac{\partial \eta_{\psi}}{\partial \phi}=0, \frac{\partial \eta_{\psi}}{\partial t}=0$, $\frac{\partial \eta_{\psi}}{\partial \theta}=0, \frac{\partial \eta_{\phi}}{\partial \psi}=0, \frac{\partial \eta_{\phi}}{\partial \phi}=0, \frac{\partial \eta_{\phi}}{\partial t}=0, \frac{\partial \eta_{\phi}}{\partial \theta}=0$ $\frac{\partial \xi}{\partial \psi}=0, \frac{\partial \xi}{\partial \phi}=0, \frac{\partial \xi}{\partial \theta}=0, \frac{\partial \xi}{\partial t}=0$ e $\eta_{\theta}=0$. Resolvendo estas equações é possível encontrar os seguintes geradores infinitesimais para a equação (9) listados na tabela (1).

O gerador infinitesimal $\mathcal{X}_{1}$ está correlacionado com a translação temporal e corresponde ao invariante associado a conservação de energia, desde que não haja dissipação. O segundo e o terceiro gerador infinitesimal correspondem à rotação $\psi$ e $\phi$ que estão diretamente ligados à conservação dos momentos angulares associados às variáveis $\psi$ e $\phi$. Para demonstrar e verificar essas constantes, os geradores infinitesimais determinados pelas simetrias de Lie, podem ser aplicados ao teorema de Noether.

\section{Teorema de Noether}

O teorema que mostra a correspondência entre as simetrias variacionais comuns e as leis de conservação dos sistemas de equações de Euler-Lagrange, foi enunciado pela Emmy Noether no início do século XX. Uma vez que admitimos simetrias de um sistema, o teorema de Noether fornece uma correspondência um-para-um entre simetrias variadas e leis de conservação 24,30 . Ou seja, para cada simetria encontrada em um sistema de equações diferenciais há uma quantidade se conservando. A busca por existência de simetrias na natureza tem como principal resultado a obtenção de invariantes. Nos casos onde se conhece $\mathcal{L}$ e as funções da transformação $\xi$ e $\eta_{q}$, a aplicação do teorema de Noether é como se fosse um revelador de quantidades $\mathcal{A}$ se conservando [50,51].

A quantidade se conservando a partir de uma transformação de simetria pode ser determinada pelo teorema de Noether a partir de 24 :

$$
\mathcal{A}=\frac{\partial \mathcal{L}}{\partial \dot{q}}\left(\dot{q} \xi-\eta_{q}\right)-\mathcal{L} \xi=\text { Constante }
$$

de forma generalizada com $n$ coordenadas como é o caso do pião, implica no seguinte invariante [52,53]:

$$
\mathcal{A}_{i}=\sum_{i=1}^{n} \frac{\partial \mathcal{L}}{\partial \dot{q}_{i}}\left(\dot{q}_{i} \xi_{i}-\eta_{q_{i}}\right)-\mathcal{L} \xi_{i} \quad \Rightarrow \quad \frac{d}{d t}(\mathcal{A})=0
$$

O resultado generaliza e unifica de uma forma que é possível ter a visualização geométrica dos princípios de conservação de energia, momento linear e momento angular. Com isso, aplicando os geradores infinitesimais encontrados pelas simetrias de Lie no teorema de Noether, equação 26), pode-se encontrar constantes de movimento. Para o primeiro gerador infinitesimal $\mathcal{X}_{1}$, sendo $\xi=1 \mathrm{e}$ $\eta_{\theta}=0$ tem-se:

$$
\begin{aligned}
& \mathcal{A}_{1}=\frac{\partial \mathcal{L}}{\partial \dot{\theta}}\left(\dot{\theta} \xi-\eta_{\theta}\right)-\mathcal{L} \xi=\frac{1}{2} I\left(\dot{\theta}^{2}+\dot{\psi}^{2} \operatorname{sen}^{2} \theta\right) \\
& +\frac{1}{2} I_{z_{2}}(\dot{\phi}+\dot{\psi} \cos \theta)^{2}+m g \ell \cos \theta
\end{aligned}
$$

que é um constante de movimento que está relacionada diretamente com a energia total do sistema, que neste caso fica sendo a energia cinética $\mathcal{T}$ mais a energia potencial $\mathcal{V}$, não havendo dissipação de energia.

Considerando agora os geradores infinitesimais $\mathcal{X}_{2}$ sendo $\xi=0$ e $\eta_{\psi}=1$ e $\mathcal{X}_{3}$ sendo $\xi=0$ e $\eta_{\phi}=1$, tem-se:

$$
\begin{aligned}
& \mathcal{A}_{2}=\frac{\partial \mathcal{L}}{\partial \dot{\psi}}\left(\dot{\psi} \xi-\eta_{\psi}\right)-\mathcal{L} \xi=I \dot{\psi} \operatorname{sen}^{2} \theta \\
& +I_{z_{2}} \cos \theta(\dot{\phi}+\dot{\psi} \cos \theta)
\end{aligned}
$$




$$
\mathcal{A}_{3}=\frac{\partial \mathcal{L}}{\partial \dot{\phi}}\left(\dot{\phi} \xi-\eta_{\phi}\right)-\mathcal{L} \xi=I_{z_{2}}(\dot{\phi}+\dot{\psi} \cos \theta)
$$

mostrando assim que as quantidades $\mathcal{A}_{2}$ e $\mathcal{A}_{3}$ estão diretamente relacionadas com os momentos angulares se conservando para a coordenada associada ao spin e com o momento também se conservando para a coordenada associada a precessão, respectivamente.

\section{Redução de Ordem e Solução}

A partir das simetrias de Lie encontradas, pode-se reduzir a ordem do sistema de equações do movimento. Por já estarem na forma canônica, serão utilizados primeiramente os geradores infinitesimais $\mathcal{X}_{2}$ e $\mathcal{X}_{3}$. Com isso, pode-se transformar a partir de uma mudança de variável, sendo $\dot{\psi}=\lambda$ e $\dot{\phi}=\zeta$ que para precessão estacionária, são constantes. Assim, a equação (9) pode ser reescrita como:

$$
\begin{aligned}
& I\left(\ddot{\theta}-\lambda^{2} \operatorname{sen} \theta \cos \theta\right)+I_{z_{2}}\left(\lambda^{2} \operatorname{sen} \theta \cos \theta+\zeta \lambda \operatorname{sen} \theta\right) \\
& -m g \ell \operatorname{sen} \theta=0 \\
& I(2 \lambda \dot{\theta} \cos \theta)-I_{z_{2}} \dot{\theta}(\lambda \cos \theta+\zeta)=0 \\
& I_{z_{2}}(\lambda \dot{\theta} \operatorname{sen} \theta)=0
\end{aligned}
$$

Para a redução de ordem de $\ddot{\theta}$, deve-se utilizar o gerador infinitesimal $\mathcal{X}_{1}$ exigindo algumas manipulações que podem ser facilitadas por programas de manipulação simbólica, como o Maple, Mathematica ou wxMaxima.

Supondo que $\mathcal{X}$ é um campo vetorial que não se anula nos pontos de origem, pode-se introduzir novas coordenadas $\left\{\bar{t}(t, \theta, \zeta, \lambda), \quad \bar{\theta}_{1}(t, \theta, \zeta, \lambda), \quad \bar{\theta}_{2}(t, \theta, \zeta, \lambda), \quad \bar{\theta}_{3}(t, \theta, \zeta, \lambda)\right\}$. A mudança de variáveis é feita usando os métodos para encontrar invariantes de grupo. Isto implica que $\mathcal{X}$ pode ser transformada na seguinte forma $\partial / \partial \bar{t}$ fornecendo assim $\bar{t}, \bar{\theta}_{1}, \bar{\theta}_{2}$ e $\bar{\theta}_{3}$ que satisfaçam as seguintes condições [30]:

$$
\begin{aligned}
& \mathcal{X}_{1}(\bar{t})=\xi \frac{\partial \bar{t}}{\partial t}+\eta_{\theta} \frac{\partial \bar{t}}{\partial \theta}+\eta_{\psi} \frac{\partial \bar{t}}{\partial \lambda}+\eta_{\phi} \frac{\partial \bar{t}}{\partial \zeta}=0 \\
& \mathcal{X}_{1}\left(\bar{\theta}_{1}\right)=\xi \frac{\partial \bar{\theta}_{1}}{\partial t}+\eta_{\theta} \frac{\partial \bar{\theta}_{1}}{\partial \theta}+\eta_{\psi} \frac{\partial \bar{\theta}_{1}}{\partial \lambda}+\eta_{\phi} \frac{\partial \bar{\theta}_{1}}{\partial \zeta}=0 \\
& \mathcal{X}_{1}\left(\bar{\theta}_{2}\right)=\xi \frac{\partial \bar{\theta}_{2}}{\partial t}+\eta_{\theta} \frac{\partial \bar{\theta}_{2}}{\partial \theta}+\eta_{\psi} \frac{\partial \bar{\theta}_{2}}{\partial \lambda}+\eta_{\phi} \frac{\partial \bar{\theta}_{2}}{\partial \zeta}=0 \\
& \mathcal{X}_{1}\left(\bar{\theta}_{3}\right)=\xi \frac{\partial \bar{\theta}_{3}}{\partial t}+\eta_{\theta} \frac{\partial \bar{\theta}_{3}}{\partial \theta}+\eta_{\psi} \frac{\partial \bar{\theta}_{3}}{\partial \lambda}+\eta_{\phi} \frac{\partial \bar{\theta}_{3}}{\partial \zeta}=1
\end{aligned}
$$

a partir dessa condição, pode-se dizer que $\bar{t}=\theta, \bar{\theta}_{1}=$ $\lambda, \bar{\theta}_{2}=\zeta$ e $\bar{\theta}_{3}=t$. Deve-se estender as mudanças de coordenadas para as derivadas de primeira ordem, então: $\dot{\bar{\theta}}_{1}=\frac{\dot{\lambda}}{\dot{\theta}}, \dot{\bar{\theta}}_{2}=\frac{\dot{\zeta}}{\dot{\theta}}$ e $\dot{\bar{\theta}}_{3}=\frac{1}{\dot{\theta}}$. Para as derivadas de segunda ordem: $\ddot{\bar{\theta}}_{3}=-\frac{\ddot{\theta}}{\dot{\theta}^{3}}$. Reescrevendo a equação (30) nas novas coordenadas e já realizando os cálculos e simplificações necessários:

$$
\begin{aligned}
& \frac{1}{2} I\left(\dot{\bar{\theta}}_{3}^{2}+\dot{\bar{\theta}}_{1}^{2} \operatorname{sen}^{2} \bar{t}\right)+\frac{1}{2} I_{z_{2}}\left(\dot{\bar{\theta}}_{2}+\dot{\bar{\theta}}_{1} \cos \bar{t}\right)^{2} \\
& +m g \ell \cos \bar{t}=\mathcal{C}_{1} \\
& I \dot{\bar{\theta}}_{1} \operatorname{sen}^{2} \bar{t}+I_{z_{2}} \cos \bar{t}\left(\dot{\bar{\theta}}_{2}+\dot{\bar{\theta}}_{1} \cos \bar{t}\right)=\mathcal{C}_{2} \\
& I_{z_{2}}\left(\dot{\bar{\theta}}_{2}+\dot{\bar{\theta}}_{1} \cos \bar{t}\right)=\mathcal{C}_{3}
\end{aligned}
$$

sendo $\mathcal{C}_{1}, \mathcal{C}_{2}$ e $\mathcal{C}_{3}$ constantes. Pode-se observar que estas equações são constantes e também são iguais às constantes de movimento obtidas anteriormente pelo teorema de Noether para este pião. Isso mostra que simetrias de Lie também são simetrias de Noether neste exemplo, como já era esperado, pois o pião é um sistema lagrangiano com equações de movimento extraídas de um princípio variacional. Por fim, pode-se determinar as expressões para $\theta, \psi$ e $\phi$. Para não perder a generalidade, utiliza-se aqui para estes cálculos, os invariantes obtidos por Noether.

A partir da constante de movimento $\mathcal{A}_{3}$, pode-se obter:

$$
\dot{\phi}=\frac{\mathcal{A}_{3}}{I_{z_{2}}}-\dot{\psi} \cos \theta
$$

Utilizando a constante $\mathcal{A}_{2}$, obtém-se:

$$
I \dot{\psi} \operatorname{sen}^{2} \theta+I_{z_{2}} \cos \theta\left(\frac{\mathcal{A}_{3}}{I_{z_{2}}}\right)=\mathcal{A}_{2}
$$

então:

$$
\dot{\psi}=\frac{\mathcal{A}_{2}-\mathcal{A}_{3} \cos \theta}{I \operatorname{sen}^{2} \theta}
$$

Introduzindo novas constantes, pode-se reescrever a constante $\mathcal{A}_{1}$ na seguinte forma:

$$
\mathcal{A}_{1}^{\prime}=\mathcal{A}_{1}-\frac{\mathcal{A}_{3}^{2}}{2 I_{z_{2}}}
$$

de onde pode-se deduzir:

$$
\mathcal{A}_{1}^{\prime}=\frac{1}{2} I \dot{\theta}^{2}+\mathcal{S}(\theta)
$$

sendo:

$$
\mathcal{S}(\theta)=\frac{\left(\mathcal{A}_{2}-\mathcal{A}_{3} \cos \theta\right)^{2}}{2 I \operatorname{sen}^{2} \theta}+m g \ell \cos \theta
$$

o potencial efetivo. Realizando uma separação de variáveis na equação (37), pode-se obter:

$$
\dot{\theta}^{2}=\frac{2}{I}\left(\mathcal{A}_{1}^{\prime}-\mathcal{S}(\theta)\right)
$$

assim:

$$
\dot{\theta}=\sqrt{\frac{2}{I}} \sqrt{\left(\mathcal{A}_{1}^{\prime}-\mathcal{S}(\theta)\right)}
$$

por fim:

$$
\int \frac{d \theta}{\sqrt{\left(\mathcal{A}_{1}^{\prime}-\mathcal{S}(\theta)\right)}}=\sqrt{\frac{2}{I}} t
$$


Não é possível expressar a solução desta integral em termos de funções elementares. Para a solução destas quadraturas podem ser usadas as integrais elípticas de Jacobi. Em um artigo recente publicado na RBEF, Lemos (2017) apresenta a importância de se utilizar as funções elípticas de Jacobi na representação deste tipo de quadratura. Muitos trabalhos e livros deixaram de abordar o uso das funções elípticas, no entanto, existem muitos problemas interessantes de mecânica que podem ser resolvidos em termos destas funções 34. O passo a passo de como se calcular uma integral utilizando as funções elípticas de Jacobi podem ser encontrados em trabalhos como o de E. Piña [54] e o próprio N. A. Lemos [34]. Com $\theta$ determinado, pode-se encontrar os outros ângulos de Euler associados ao movimento do pião.

A partir disso, pode-se utilizar a seguinte equivalência para a determinação da solução analítica do pião simétrico 34,51 :

$$
\begin{gathered}
\operatorname{sn}(u, k) \equiv \operatorname{sen} \bar{\theta}=\operatorname{sen}(\operatorname{am} u) \\
\operatorname{cn}(u, k) \equiv \cos \bar{\theta}=\cos (\operatorname{am} u) \\
\operatorname{dn}(u, k) \equiv \sqrt{1-k^{2} \operatorname{sn}^{2} u}=\frac{d(\operatorname{am} u)}{d u}
\end{gathered}
$$

sendo am a função amplitude e $k$ o seu módulo, sn a função seno elíptico de Jacobi e cn a função cosseno elíptico. A partir das definições trigonométricas, pode-se obter as seguintes identidades com as funções elípticas:

$$
\begin{gathered}
\operatorname{sn}^{2} u+\operatorname{cn}^{2} u=1 \\
\operatorname{dn}^{2} u+k^{2} \operatorname{sn}^{2} u=1 \\
\operatorname{cn}^{2} u+\left(1-k^{2}\right) \operatorname{sn}^{2} u=\operatorname{dn}^{2} u
\end{gathered}
$$

Com a consideração do argumento $(u, k)$ na transformação obtém-se finalmente a solução analítica para as equações de movimento do pião simétrico:

$$
\begin{gathered}
\theta(t)=\operatorname{sgn}(a+b)\left[\sqrt{1-c^{2}} \operatorname{dn}(u, k) \operatorname{sen}(\Omega)\right. \\
+(d-c) \operatorname{cn}(u, k) \operatorname{sn}(u, k) \cos (\Omega)] \\
\psi(t)=-\sqrt{1-c^{2}} \operatorname{dn}(u, k) \cos (\Omega) \\
+(d-c) \operatorname{cn}(u, k) \operatorname{sn}(u, k) \operatorname{sen}(\Omega) \\
\phi(t)=c+(d-c) \operatorname{sn}^{2}(u, k)
\end{gathered}
$$

sendo $a=\frac{\mathcal{A}_{3}}{I}, b=\frac{\mathcal{A}_{2}}{I}, c=\frac{\cos \theta}{\operatorname{sn}^{2}(u, k)}, d=\frac{b}{a}, \Omega=$ $\frac{\operatorname{cn}(u, k)}{\operatorname{sn}(u, k) \operatorname{dn}(u, k)}$ e sgn a função sign:

$$
\operatorname{sgn}(a+b)=\left\{\begin{array}{lll}
-1 & \text { se } & a+b<0 \\
0 & \text { se } & a+b=0 \\
1 & \text { se } & a+b>0
\end{array}\right.
$$

\section{Conclusões}

Observa-se que a obtenção de simetrias de Lie podem ser usadas de forma efetiva na redução de ordem e obtenção de integrais primeira que facilitam a integração das equações de movimento em problemas de mecânica clássica. Neste sentido, é possível ilustrar e apresentar a teoria de Lie usando o movimento de um pião simétrico, comumente estudado nos primeiros anos de cursos de mecânica. Os requisitos matemáticos para tal, usam conceitos de cálculo de várias variáveis que são comuns a todos os estudantes de física, matemática ou engenharia de anos iniciais. A partir das simetrias de Lie extraídas de equações determinantes pode-se também relacioná-las diretamente com os teoremas clássicos de conservação. Uma comparação com as constantes de movimento extraídas pelo Teorema de Noether também é feita neste sentido. A partir disto, usam-se as simetrias de Lie para gerar transformações visando a solução analítica completa das equações de movimento do pião em precessão estacionária, empregando para isto as funções elípticas de Jacobi.

\section{Agradecimentos}

O primeiro autor agradece a CAPES por sua bolsa de doutorado e de sanduíche na University of Minnesota (USA). Os autores também agradecem o apoio financeiro do Edital Universal CNPq - Processo 404463/2016-9 e as discussões sobre Simetrias de Lie com o Prof. Peter J. Olver da University of Minnesota. Adicionalmente, os autores gostariam de agradecer os revisores anônimos e o Editor Associado pelos seus comentários e sugestões úteis e relevantes.

\section{Referências}

[1] J. Roychowdhury, IEEE Transactions on Circuits and Systems I: Fundamental Theory and Applications 48, 578 (2001).

[2] N. Schunck, J. Dobaczewski, J. McDonnell, W. Satula, J. A. Sheikh, A. Staszczak, M. Stoitsov and P. Toivanen, Computer Physics Communications 183, 166 (2012).

[3] A. Akbulut and F. Taşcan, Chaos, Solitons and Fractals 100, 33 (2017).

[4] P.J. Olver, Journal of Mathematical Analysis and Applications 333, 450 (2007).

[5] D.J. Arrigo, Symmetry Analysis of Differential Equations: An Introduction (John Wiley \& Sons, Conway, 2015).

[6] V.M. Boyko, R.O. Popovych and N.M. Shapoval, Journal of Mathematical Analysis and Applications 397, 434 (2013).

[7] PP. Cai, JL. Fu and YX. Guo, Reports on Mathematical Physics 79, 279 (2017).

[8] B.J. Cantwell, Introduction to Symmetry Analysis, (Cambridge University Press, Cambridge, 2002).

[9] F. Caruso, Revista Brasileira de Ensino de Física 30, 3309 (2008). 
[10] A. Mouchet, Annals of Physics 372, 260 (2016).

[11] D.M. Ambrose, J.P. Kelliher, M.C. Lopes Filho and H.J.N. Lopes, Journal of Differential Equations 259, 4509 (2015).

[12] P.A. Clarksonz and E.L. Mansfield, Physica D: Nonlinear Phenomena 70, 250 (1994).

[13] A. Paliathanasis and M. Tsamparlis, Journal of Geometry and Physics 62, 2443 (2012).

[14] I.V. Stepanova, Communications in Nonlinear Science and Numerical Simulation 20, 684 (2015).

[15] M. Craddock, Journal of Differential Equations 166, 107 (2000).

[16] M.T. Mustafa and A.Y. Al-Dweik, Communications in Nonlinear Science and Numerical Simulation 23, 141 (2015).

[17] C.-S. Liu, International Journal of Non-Linear Mechanics 69, 93 (2015).

[18] T. Bartsch and Y. Ding Nonlinear Analysis: Theory, Methods and Applications 44, 727 (2001).

[19] J. Bocko, V. Nohajová and T. Harcarik Procedia Engineering 48, 40 (2012).

[20] A.E. Santana, A. Ribeiro Filho and J.D.M. Vianna, Revista Brasileira de Ensino de Física 22, 199 (2000).

[21] J-L. Fu and L-Q. Chen, Mechanics Research Communications 31, 9 (2004).

[22] Y. Kosmann-Schwarzbach, The Noether Theorems: Invariance and Conservation Laws in the Twentieth Century, (Springer-Verlag, New York, 2011).

[23] L-L. Feng, S-F. Tian, T-T. Zhang and J. Zhou, Chinese Journal of Physics 55, 996 (2017).

[24] A. de Souza Martins, Revista Brasileira de Ensino de Física 21, 33 (1999).

[25] I.L. Freire, P.L. da Silva and M. Torrisi, Journal of Physics A: Mathematical and Theoretical 46, 245206 (2013).

[26] I.L. Freire and P.L. da Silva, in Anais do Congresso de Matemática Aplicada e Computacional, Bauru, (2013), 622.

[27] X-H. Zhai, Y. Zhang and F. Mei, Communications in Nonlinear Science and Numerical Simulation 52, 32 (2017).

[28] J-L. Fu and L-Q. Chen, Physics Letters A, 317, 9 (2003).

[29] Y.Y. Zhao, Acta Mechanica Sinica 26, 380 (1994).

[30] P.J. Olver, Applications of Lie Groups to Differential Equations (Springer, New York, 1986).

[31] P.G. Estévez, F.J. Herranz, J. de Lucas and C. Sardón, Applied Mathematics and Computation 273, 435 (2016).

[32] N. Martins and D.F.M. Torres, Applied Mathematics Letters 23, 1432 (2010).

[33] Q. Jia, H. Wu and F. Mei, Journal of Mathematical Analysis and Applications 442, 782 (2016).

[34] N.A. Lemos, Revista Brasileira de Ensino de Física 39, e1305 (2017).

[35] H. Goldstein, C. Poole and J. Safko, Classical Mechanics (Addison Wesley, Boston, 2002).

[36] J.R. Taylor, Classical Mechanics (University Science Books, South Orange, 2004).

[37] N.A. Lemos, Mecânica Analítica (Editora Livraria da Física, São Paulo, 2007).

[38] I.S. Ferreira, Dinânica de Sistemas Mecânicos (Makron Books Ltda, São Paulo, 2001).
[39] D.F. Lawden, Elliptic Functions and Applications (Springer-Verlag, New York, 1989).

[40] L.H.A. Monteiro, Sistemas Dinânicos (Editora Livraria da Física, São Paulo,, 2006).

[41] G. Baumann, Symmetry Analysis of Differential Equations with Mathematica (Springer-Verlag, New York, 2000).

[42] G.W. Blumman and S. Kumei, Symmetries and Differential Equations (Springer-Verlag, New York, 1989).

[43] S. Lie and G. Scheffers, Vorlesungen Über Differentialgleichungen Mit Bekannten Infinitesimalen Transformationen (B.G. Teubner, Leipzig, 1891).

[44] S. Lie, Arch. Math. 3, 328 (1881).

[45] G.W. Blumman, Journal of Mathematical Analysis and Applications 145, 52 (1990).

[46] F.M. Mahomed and P.G.L. Leach, Journal of Mathematical Analysis and Applications 151, 80 (1990).

[47] J.E. Marsden, and T.S. Ratiu, Introduction to Mechanics and Symmetry: A Basic Exposition of Classical Mechanical Systems (Springer-Verlag, New York, 1999).

[48] R. McWeeny, Symmetry: An Introduction to Group Theory and its Applications (Dover, New York, 2002).

[49] S. Dimas andD. Tsoubelis, in: Proceedings of the 10th International Conference in Modern Group Analysis, Larnaca, (2004), 64.

[50] F.X. Mei, Acta Mechanica 141, 135 (2000).

[51] A.J. Brizard, Classical Physics 1, 1 (2007).

[52] N.A. Lemos, Revista Mexicana de Física 39, 304 (1993).

[53] E. Noether, Göttingen Nachrichten 1, 37 (1918).

[54] E. Piña, European Journal of Physics 38, 035001 (2017). 